\title{
Effect of cannabinoids intake on increasing appetite and change of eating behavior
}

\author{
A. Skawiańczyk ${ }^{1}$, P. Walecki , S. Gryboś ${ }^{1}$, A. Zwierzchowska1, \\ E.J. Gorzelańczyk²
}

1 Jagiellonian University Medical College, Faculty of Medicine, Krakow, Poland

2 Kazimierz Wielki University, Laboratory of Cognitive Science Institute of Philosophy, Bydgoszcz, Poland.

\section{OBJECTIVES}

The aim of this study is the assessment of the effect of exogenous cannabinoids on eating behavior.

In recent years, the role of cannabinoids in food intake associated with the activation of the brain reward system has again become interested. This indicates the potential use of the endocannabinoid system in the treatment of diseases with appetite disorders and neurodegenerative, debilitating diseases. The endocannabinoid system (ECS) plays a significant role in the regulation of energy consumption. Due to two pathways of influence - one related to the regulation of energy balance under the control of leptin hormone and the other related to the hedonic aspect of eating. Thanks to its properties, the ECS can be an attractive therapeutic target in the treatment of diseases associated with loss of appetite. Both exogenous cannabinoids, which are active components of Cannabis spp., and endogenous cannabinoids, such as anandamide and 2-AG, have been associated with appetite stimulating properties. The feeling of increased appetite after using cannabis is referred to as 'the munchies'.

\section{METHODS}

Twenty drug-dependent people taking cannabinoids were enrolled in the study. Sixteen people had the correct body mass index (BMI), between 18.5 and 24.9. In two people BMI reached the value of being overweight. One person was underweight (BMI was below 18.5).

Each examined person provided ten 24-hour dietary recall interviews: five of days in which cannabinoids were used and five days when participants did not use cannabinoids. In total, one hundred and eighty 24hour interviews were collected. Nutrients were calculated on the basis of the Polish Food Composition Database and the Dieta 5.0 software.

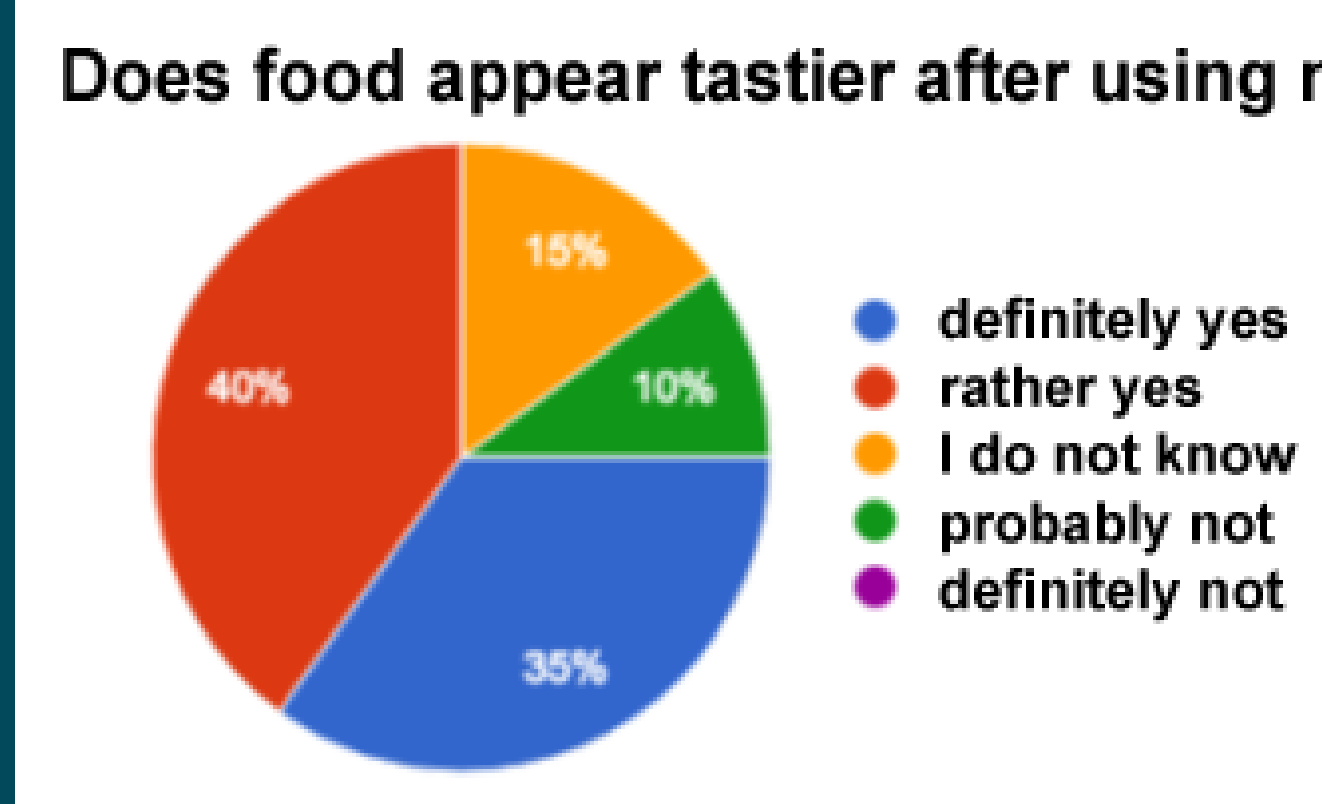

Correspondence address: askawianczyk@gmail.com

\section{RESULTS}

$75 \%$ of the people participating in the study admitted that under the influence of cannabinoids, they feel increased appetite and increased attractiveness of food, while $15 \%$ of participants declared that the cannabinoids did not change their appetite and did not change the feeling of pleasure while eating. The average amount of calories consumed on the days when participants used cannabinoids is $\mathbf{4 2} \%$ higher compared to the days when cannabinoids were not used. The amount of magnesium, zinc, manganese and vitamin $E$, vitamins B1, B2, B6, A, C retinol and carotenes delivered is significantly higher on days when participants use cannabinoids.
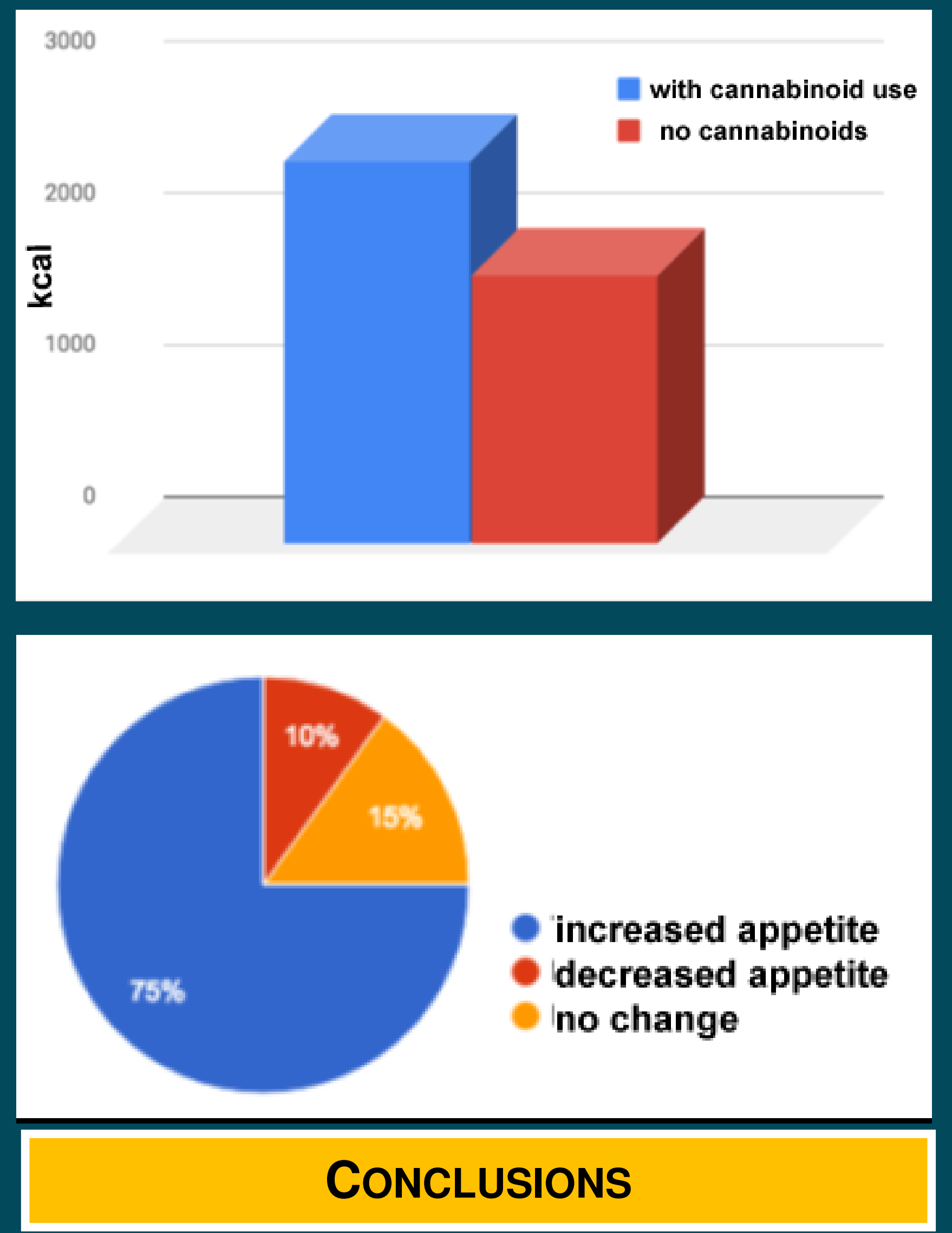

The use of exogenous cannabinoids has an impact on the amount of energy supplied, macronutrients and some micronutrients. 